\title{
IAMJ
}

INTERNATIONAL

AYURVEDIC

MEDICAL JOURNAL

\section{CONCEPTUAL STUDY ON CLINICAL APPLICATION OF YAVAGU KALPANA}

\author{
Swati Thakre', Mangesh Nalkande ${ }^{2}$ \\ ${ }^{1}$ Associate Professor, Dept of Panchakarma, Dr R N Lahoti Ayurved Medical College, Hospital and Research \\ Centre, Sultanpur, Buldhana (Maharashtra), India \\ ${ }^{2}$ Assistant Professor, Dept of Kayachikitsa, Govt Ayurved College Nanded, (Maharashtra), India
}

Corresponding Author: drawathiayurveda@gmail.com

\section{https://doi.org/10.46607/iamj5609092021}

(Published Online: September 2021)

Open Access

(C) International Ayurvedic Medical Journal, India 2021

Article Received: 30/08//2021 - Peer Reviewed: 06/09/2021 - Accepted for Publication: 07/09/2021

\section{Check for updates}

\section{ABSTRACT}

In Ayurveda many times Aushadhi Dravya's our combined with Aahariya Dravya's in different conditions where the patient is not able to consume the medicine due to their Virya Pradhanta, Bala Kshya or Agnimandhya "Yavagu Kalpna" is one of the Ahariya Kalpna which when siddha with Aushadhi Dravya's can be used in different conditions for eradication of the disease. Yavagu not only cure's the disease but also helps in the prevention of a disease by its different properties and nutritive value. So, in this review "Yavagu Kalpana" is critically analysed \& compiled from Ayurveda text for their clinical application in future.

Keywords: Yavagu, Aushadhi Dravya, Aahariya Dravya

\section{INTRODUCTION}

Ayu i.e., life is comprised of several factors like "Sharir", "Satva", indriya and "Atma", which are connected by Prana. The major stambha i.e., pillars called tristambha are Dosha's, while to sustain the life process Ayurveda has forwarded the concept of Trayopasthambha which are
1) Ahara
2) Nidra
3) Brahmanarya 
According to Charka ahara is the major source for the continuation of chetana. Anurutti or continuation of life process, man is the result of food and he is best supported by wholesome food. Aahar is called, "Mahabhaisajya" in Kashyapa Samhita. In Ayurveda many times Aushodha is advised with Aahriya dravyas in different conditions lie, vruddhavastha (old age) balyavastha (paediatrics) Grabhini (ANC), Sukumar Person, alpa bala due to chronic disease agnimandhya etc.

"Yavagu" is one of ahar kalpna which can be used for eradication of disease as well as for the nutritive need of our body. It is used as pathya kalpna in various conditions for the prevention of disease. Due to its different properties, it has a wide range of actions in the alleviation of different vikara. So, in this review, "Yavagu Kalpna" is critically analysed from different Ayurvedic texts.

Aim:

1) To study the Yavagu kalpna.
2) To compile the different Yavagu kalpna \& its clinical application from text.

\section{Yavagu Kalpna: -}

Yavagu Kalpna is one of ahar Kalpna which is included in Pathya Kalpna. It is prepared using rice, pulses and water. It is a semisolid gruel having a lot of nutritive value. It is used as an ahar and as well as aushadhi.

\section{Classification of ago: -}

Yavagu are broadly classified as

i) On Basis of Constituents:

On basis of its constituents Yavagu is classified as:

1) Aahar Pradhan

2) Aushadhi Pradhan

ii) On basis of Bhaisjya Kalpna

On basis of Bhaisjya Kalpna Yavagu is classified as:

1) Kalka pradhan

2) Kwath pradhan

3) Mamsras pradhan

Table 1

\begin{tabular}{|l|l|l|l|}
\hline Sr.No. & Yavagu type & Constituents & Uses \\
\hline 1 & $\begin{array}{l}\text { Aahar Pradhan (Called Raspradhan } \\
\text { Yavagu) }\end{array}$ & $\begin{array}{l}\text { Ahar Draya like Tandula Masha, } \\
\text { Tila, etc. }\end{array}$ & $\begin{array}{l}\text { Used as pathya in healthy person for } \\
\text { preventive purpose }\end{array}$ \\
\hline 2 & $\begin{array}{l}\text { Aushadhi Pradhan (Called Virya } \\
\text { Pradhan Yavagu) }\end{array}$ & $\begin{array}{l}\text { Aushadhi Dravya as well as as } \\
\text { aharia draya }\end{array}$ & Used in many diseased conditions. \\
\hline
\end{tabular}

Table 2

\begin{tabular}{|c|c|c|c|}
\hline Sr.No. & Yavagu type & Constituents & Uses \\
\hline 1 & Kalka Pradhan & Yavagu prepared with kalka & $\begin{array}{l}\text { Eq. Sunthi, pipalli siddha in udar } \\
\text { shula }\end{array}$ \\
\hline 2 & Kwath pradhan & Yavagu prepared with kwath & Eq. Bilva kwath siddha in atisara \\
\hline 3 & Mamsrasa Pradhan & 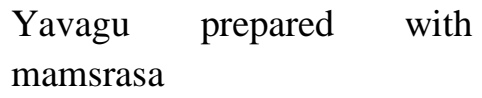 & $\begin{array}{l}\text { Eq. Mamsrasa siddha in karshya } \\
\text { kshya }\end{array}$ \\
\hline
\end{tabular}

Ausadhi pradhan Yavagu is further classified as.

Table 3

\begin{tabular}{|l|l|l|}
\hline Sr.No. & Yavagu & Uses \\
\hline 1 & Tikshana Virya & For eg. Pippali sunthi in shula \\
2 & Madhyam Virya & eg. Bilva siddha in Atisara \\
3 & Mrudu Virya & eg. Amalki siddha \\
\hline
\end{tabular}


Aacharya Vagbhatta has not used the specific word Yavagu. He has described ahar kalpna in kruttana Varga (Ref. AH. St. 6 / 26-27)

He has classified it as: -

1) Manda

2) Peya

3) Vilepi

4) Odana

Manda: - Thin fluid resembling water drain out immediately after cooking is known as Manda.

Peya: - Slightly thicker than manda but still liquid is peya.

Vilepi: - Next stage with more solid grain 8 less liquid is vilepi.
Odana: - The last stage which is solid without fluid part is odana.

On this Arundatta comments that peya 8 vilepi both are included in Yavagu Kalpna. They can be the types of Yavagu Kalpna. He describes Mand as "asikhto dravya" 8 Yavagu as. "Sikhto Dravya" which are of two types.

i) Peya - alpa sikhto

ii) Vilepi - Bahu sikhto And Odana as "Adravyani Sikhtani"

So, from the above explanation peya \& vilepi can be considered as a type of Yavagu on basis of consistency.

\section{Table 4}

\begin{tabular}{|l|l|l|l|}
\hline Sr.No. & Name of Kalpna & Rice proportion & Water proportion \\
\hline 01 & Manda & 1 part & 14 parts \\
\hline 02 & Peya & 1 Part & 14 Parts \\
\hline 03 & Vilepi & 1 Part & 4 Parts \\
\hline 04 & Odana & 1 Part & 5 Parts \\
\hline
\end{tabular}

Method of preparation of Yavagu: -

i) Constituents of Yavagu: - Acharya Sushrut and sharangdhara have a different opinions regarding constituents of Yavagu.

Table 5

\begin{tabular}{|l|l|l|l|}
\hline Sr.No. & Text & Constituents & Properties \\
\hline 1 & $\begin{array}{l}\text { Sharangdhara } \\
\text { S.S. Mkh-2/164-/65 }\end{array}$ & $\begin{array}{l}\text { 1) Tandula (Oryza-sativa-linn) } \\
\text { 2) Mugda (Phaesolus.mungo. linn) } \\
\text { 3) Masha (Phaesolusradiatus.linn) } \\
\text { 4) Tila (Seasum. indicum) }\end{array}$ & $\begin{array}{l}\text { Balya, Grahi } \\
\text { Trapak } \\
\text { Vatanashaka }\end{array}$ \\
\hline 2 & $\begin{array}{l}\text { Sushrut } \\
\text { (S.S. Utt 40/156) }\end{array}$ & 1) Tandula (Oryza-sativa. linn) & $\begin{array}{l}\text { Laghu, Grahi, } \\
\text { Bastishodhani }\end{array}$ \\
\hline
\end{tabular}

\section{II) Preparatory Procedure: -}

i) Procedure for Aahar Siddha Yavagu: -

For ahar Siddha Yavagu 1 part of ahar dravya (i.e., trindula masha etc) is cooked with 6 parts of water to get a semisolid gruel.

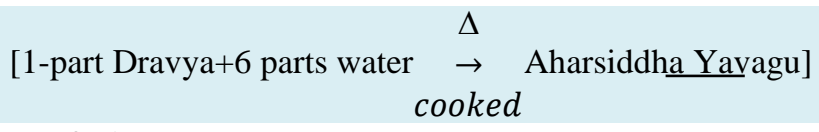

(Ref.Sh.S.MK.2/164-165)

\section{ii) Procedure for Aushadhi Siddha Yavagu: -}

For Aushadhi Siddha Yavagu 4 Pal of aushadhi dravya (e.g. Sunthi Pippali etc) is boiled with 64 pal of water and reduced to half a quantity and then to this rice is added and cooked to get a semisolid gruel.

(Ref. Sh.S.MK.2/152)

This is a general method for preparation of aushadhi Siddha Yavagu but wherever special proportion is mentioned that must be followed. 


\section{Review of some classical preparation of Yavagu:}

Let us review some classical preparation of Yavagu and its clinical applications.

\begin{tabular}{|c|c|c|c|c|}
\hline Sr.No. & Name of Yaugu & Contents of Yavagu & Properties and indication & Reference \\
\hline 1 & Agnideepak yavagu & $\begin{array}{l}\text { Pippali (Pipper congum) } \\
\text { Pippalimula } \\
\text { Chava (piper chaba) } \\
\text { Chitraka (Plumbago Zeybuica) } \\
\text { Sunthi ((Zingiber officihale) }\end{array}$ & $\begin{array}{l}\text { Agnimandhya } \\
\text { Shula }\end{array}$ & C.S. Su2/18 \\
\hline 2 & $\begin{array}{l}\text { Pachani \& } \\
\text { Yavagu Grahini }\end{array}$ & $\begin{array}{l}\text { Kapitha (Feroria limonia) } \\
\text { Bilva (Aegles marmelos) } \\
\text { Changeri (Oxalis Corniculata) } \\
\text { Dadim (Punica-granature) } \\
\text { Dadhi (curd) }\end{array}$ & Atisara & $\begin{array}{l}\text { C.S.SU 2/19 } \\
\text { A.S. Ch 11/14 }\end{array}$ \\
\hline 3 & $\begin{array}{l}\text { Pitta Kaphaj } \\
\text { Atisara Nashak }\end{array}$ & $\begin{array}{l}\text { Hiber (Coleus velliveroides) } \\
\text { Utpal (Nymphaeoallra) } \\
\text { Musta (Cyperus rotundus) } \\
\text { Prushparui (Uraria Picta) } \\
\text { Dadhim (Punica granatum) }\end{array}$ & $\begin{array}{l}\text { Pittaj Kaphaj } \\
\text { Atisarsa }\end{array}$ & C.S.S.2/10 \\
\hline 4 & Rakataj Atisar Nashak & $\begin{array}{l}\text { Hiber (Colecus vellivenoides) } \\
\text { Utpal (Nymphaeoallra) } \\
\text { Musta (Cypews rotundus) } \\
\text { Prushparui (Uraria Picta) } \\
\text { Chhag. ksheer (Goat milk) }\end{array}$ & Raktaj Atisara & $\begin{array}{l}\text { C.S.SU } 2 / 21 \\
\text { A.H.C. } 9 / 86\end{array}$ \\
\hline 5 & $\begin{array}{l}\text { Aamatisar } \\
\text { Nashak Yavagu }\end{array}$ & $\begin{array}{l}\text { Ativisha (Aconitum heterophylum) } \\
\text { Sunthi (zingiber Officinale) } \\
\text { Dadim (Punicagranatum) }\end{array}$ & Aamatisara & C.S.SU-2/22 \\
\hline 6 & $\begin{array}{l}\text { Pakratisara Nashak Ya- } \\
\text { vagu }\end{array}$ & $\begin{array}{l}\text { Bilva (Aegelos marmelos) } \\
\text { Musta (Cyperus rotondus) } \\
\text { Dhatki (Wood fordia fruticosa) } \\
\text { Sunthi (Zingiber officinale) }\end{array}$ & Pakvatisara & AH.C.9/23-24 \\
\hline 7 & Kriminashak Yavagu & $\begin{array}{l}\text { Vidanga (Embelica ribes) } \\
\text { Pippalimula (Pipper longum) } \\
\text { Shigru (Moringa pterygosperma) } \\
\text { Marich (Piper nigrum) } \\
\text { Takra (butter milk) }\end{array}$ & Krimiroga & $\begin{array}{l}\text { C.S.SU } 2 / 23 \\
\text { AH.C. } 20 / 25\end{array}$ \\
\hline 8 & Trishnashmak yavagu & $\begin{array}{l}\text { Sariva } \\
\text { Musta (Cyperus rotandus) } \\
\text { Pippali (Pipper longum) } \\
\text { Mudvika, Lajja \& madhu }\end{array}$ & Trishna roga & C.S.SU $2 / 224$ \\
\hline 9 & Vishghani Yavagu & Somraji & Vishvikara & C.S.SU $2 / 24$ \\
\hline 10 & Bruhaniya Yavagu & $\begin{array}{l}\text { Mamsrasa (Meat soup) } \\
\text { (Varha Mamsa) }\end{array}$ & $\begin{array}{l}\text { Krishata } \\
\text { Kshya } \\
\text { Kshata }\end{array}$ & C.S.SU $2 / 25$ \\
\hline 11 & Karshaniya Yavagu & Gavedhuk siddha yavagu & Sthodlya Medoroga & C.S.SU $2 / 25$ \\
\hline 12 & Shothahar Yavagu & Dashmula, yava, shali Lavana \& Sneha & Shotha & A.H.C. $17 / 17$ \\
\hline 13 & Snehanartha Yavagu & $\begin{array}{l}\text { Tila (Seacum Indicum) } \\
\text { Ghrita (ghee) Lavana (sact) }\end{array}$ & For Snehan & C.S.SU 2/26 \\
\hline 14 & Rukshanartha Yavagu & Kushamula & For & C.S.SU $2 / 26$ \\
\hline
\end{tabular}




\begin{tabular}{|c|c|c|c|c|}
\hline & & Amalaki (Embelica officinalis) & Rukshan & \\
\hline 15 & $\begin{array}{l}\text { Kasashwas Nashak Ya- } \\
\text { vagu }\end{array}$ & $\begin{array}{l}\text { 1) Dashmula siddha yavagu } \\
\text { 2) Pippali (Pipper longum) } \\
\text { Ajmoda (carum roxburghinum) } \\
\text { Bilva (Aeqles marmelocus) } \\
\text { Sunthi (zinziberofficinale) } \\
\text { Jwavanashak Dhanyaka (coriander sa- } \\
\text { tivum) }\end{array}$ & $\begin{array}{l}\text { Hiccha } \\
\text { Shwas } \\
\text { kasa } \\
\text { Jawara } \\
\text { Trishna }\end{array}$ & $\begin{array}{l}\text { C.S.SU } 2 / 27 \\
\text { AH Chi.3/20- } \\
21 \\
\text { A.S. Chi } 24 / 25\end{array}$ \\
\hline 16 & Rechak yavagu & $\begin{array}{l}\text { Mash (Phaseocusmango) } \\
\text { Tila (Seasum Indicum) } \\
\text { Shaka, Mamsarasa }\end{array}$ & Rechan & C.S. SU $2 / 28$ \\
\hline 17 & Grahi Yavagu & $\begin{array}{l}\text { Jombu (Syzgum cumini) } \\
\text { Bilwa (Aeglemarmelos) } \\
\text { Amra (Mangifera indica) }\end{array}$ & Mala sangrahi & $\begin{array}{l}\text { C.S.SU } 2 / 28 \\
\text { A.S. Chi } 11 / 14\end{array}$ \\
\hline 18 & Bhedani Yavagu & $\begin{array}{l}\text { Chitraka (Plumbagozeylanica) } \\
\text { Amlavetsa (Gorcinia pendunculata) } \\
\text { Hingu (Ferula foetida) } \\
\text { Kshara }\end{array}$ & Bhedhan & C.S.SU $2 / 29$ \\
\hline 19 & Vatanulomana yavagu & $\begin{array}{l}\text { Haritkai (terminalia chebula) } \\
\text { Pippalimula (piper longum) } \\
\text { Vishwa (Zingiber officinale) }\end{array}$ & Vatanulomna & C.S.SU $2 / 29$ \\
\hline 20 & Updravnashak yavagu & Takra (buttermilk) & Sneha vyapad & C.S.SU $2 / 30$ \\
\hline 21 & $\begin{array}{l}\text { Vishmajwar Nashak } \\
\text { Yavagu }\end{array}$ & Gavya Mamsa, Dadim & Vishamjwara & C.S.SU $2 / 31$ \\
\hline 22 & Kantharoga Nashak & $\begin{array}{l}\text { Yavachurna (Hordeum vulgare) Ghrita \& } \\
\text { Talia }\end{array}$ & Kantha Roga & C.S. SU $2 / 31$ \\
\hline 23 & Udarroga Nashak & Tandula, Gomutra Ksheer & Udar Roga & A.H.C. $15 / 23$ \\
\hline 24 & Kaphodara nashak & $\begin{array}{l}\text { Sunthi (Zinxiberofficinale) } \\
\text { Maricha (Piper nigrum) } \\
\text { Pippali (pipper longum) } \\
\text { Yavakshara (Potasli carbonas) }\end{array}$ & Kaphodara & A.S. Chi $17 / 58$ \\
\hline 25 & $\begin{array}{l}\text { Aamalika Siddha Ya- } \\
\text { vagu }\end{array}$ & $\begin{array}{l}\text { Aamalki (Embelica officinalis) } \\
\text { Sunthi (zinxiber officinale) } \\
\text { Khandasharkara }\end{array}$ & $\begin{array}{l}\text { Asweda } \\
\text { Anidra }\end{array}$ & A.H. Chi $1 / 3.2$ \\
\hline 26 & Chavyadi Yavague & $\begin{array}{l}\text { Chavya (piper chalra) } \\
\text { Twak (Cinnamomium zelyanicum) } \\
\text { Pipalimula (piper lorgum) } \\
\text { Dhataki (wookd fordia frutcosa) } \\
\text { Shunthi (zingiber officinale) } \\
\text { Marich (piper longum) } \\
\text { Chitrak (plumbago zeylanica) } \\
\text { Kapitha (feronia limonia) } \\
\text { Bilva (aegles marmelos) } \\
\text { Patha (cissampelos pareira) } \\
\text { Shalmali (salmalia malabrica) }\end{array}$ & Crahauni & C.Chi $15 / 113$ \\
\hline 27 & Dhatakyadi Yavagu & $\begin{array}{l}\text { Musta (cyperus rotandus) } \\
\text { Dhataki (woofordia fruitcose) } \\
\text { Bilva (Aegle marmelos) }\end{array}$ & Raktapitha & C, Chi $4 / 46$ \\
\hline
\end{tabular}




\begin{tabular}{|c|c|c|c|c|}
\hline & & Drualabha (fogonia Arabica) & & \\
\hline 28 & Jivantyadi Yavagu & $\begin{array}{l}\text { Jivanti (Leptadenia reticulate) } \\
\text { Ajgi (Cuminum cyminum) } \\
\text { Shati (Hedychium spicatum) } \\
\text { Chitraka (plumbago zeylanica) } \\
\text { Pushkarmula (Amula racemosa) } \\
\text { Karavi (corcum carvi), Bilva. } \\
\text { Vrukshmala (Gracinia indica) } \\
\text { Dashmula, Ghrita, Taila and Yavakshara }\end{array}$ & Shotha & $\begin{array}{l}\text { C.Chi } 12 / 60 \\
\text { A.H. Chi } 11 / 20\end{array}$ \\
\hline 29 & Mutrala Yavagu & Kukkuta Mamsarasa & Mutrala & A.H. Chi $3 / 47$ \\
\hline
\end{tabular}

\section{DISCUSSION}

From the above review we can say that Yavagu has wide range of actions:

- Yavagu corsets Ama dosha by their laghu, ushna properties and by agnideepan in nature

- Dosha which is gradually accumulated and adherent in srotas become loose and get easily separated by laghu dravya, ushna and sartva properties of yavagu.

- Aushadhi dravya's in yavagu kalpna are vyadhi pratyanik in nature but they also help in samprapti vighatana of vyadhi.

- They cause sweating (swedon) because of their ushnatva

- They alleviate thirst because of their Dravatva (liquid nature)

- They sustain life (Ayushakara) due to their nutritional value.

- They are easily digestible because of their fluidity and permeability.

- As a result, they are easily absorbed in the body and provide instant energy i.e., Balya in nature.

- They are shulghna, angnideepak, anulomak krimighna, vishghna, jwarghna, mutrala vatanashaka, bastishodhak, Grahi in action, so used in different vyadhis like Jwar, shwas, kasa, udara, shotha atisara etc.

So, keeping in mind the nature of the body, Kala i.e., ritu (season) and strength of a patient-physician can advise yavagu kalpna in their practice.

\section{CONCLUSION}

By this review study, it can be concluded that yavagu is the combined Kalpna of ahar and aushadhi which can be helpful in both healthy and diseased conditions. So, we the physician should incorporate the use of these different Yavagu kalpna's in our daily practice to achieve the aim of Ayurveda i.e.

"Swasthasya Swastha Rakshanam Aaturasaya Vyadhi Parimoksha..."

\section{REFERENCES}

1. Acharya Chanaka, Charka Samhita, Shastri K, Chaturvedi G. Comm. Chaukhamba sharti Academy Reprinted 2008.

2. Vruddhajeevaka, Kashyapa Samhita, hemraj Sharma editor $9^{\text {th }}$ Edition 2004 Khilastana

3. Vagghatta Ashtang Hridayam (Sarvanga sundara and Ayurveda Rasayana commentary)

4. Acharya Sharangdhara, Sharangdhar Samhita $4^{\text {th }}$ edition chaukhamba prakashan $4^{\text {th }}$ edition.

5. Acharya, Sushrut Samhita, Ambika Datta, Shastri editor reprint 2010 uttarsthana.

\section{Source of Support: Nil \\ Conflict of Interest: None Declared}

How to cite this URL: Swati Thakre \& Mangesh Nalkande: Conceptual Study On Clinical Application Of Yavagu Kalpana. International Ayurvedic Medical Journal \{online\} 2021 \{cited September 2021\} Available from: http://www.iamj.in/posts/images/upload/2279 2284.pdf 